$t_{L_{1}} \quad=$ Laufzeit der ersten Teilchensorte

$t_{L_{2}}=$ Laufzeit der zweiten Teilchensorte

$t_{L_{\max }}=$ Laufzeit der langsamsten Teilchensorte

$t_{L_{\min }}=$ Laufzeit der schnellsten Teilchensorte

$b_{0,5}=$ Halbwertsbreite

$L=$ Länge der Trennstrecke

$n=$ Anzahl der „Trennstufen“

$\alpha=$ Laufzeitverhältnis zweier Teilchensorten

$\varepsilon \quad=$ Breite der Sammelfalle $\vartheta \quad=$ Auflösung zweier Teilchensorten

$\eta \quad=$ Zähigkeit der Flüssigkeit

\section{Literatur}

1. Kaiser, R.: Chromatographie in der Gasphase, 1. Teil, S. 52. Mannheim: Bibliograph. Inst. 1960.

Dr. H. Pauschmann

Lehrstuhl für organische Chemie der Universität

74 Tübingen, Nauklerstr. 37a

\title{
Massenspektrometrischer Nachweis von Arzneimittelmetaboliten (Barbiturate, Noludar, Pyramidon) im Rahmen der forensischen Analyse
}

\author{
W. ARTold und H. F. GrÜtzMacher \\ Forensisch-toxikologische Laboratorien (Doz. Dr. Dr. W. Arnold) des Institutes für gerichtliche Medizin und Kriminalistik \\ (Direktor: Prof. Dr. W. Janssen) und Chemisches Staatsinstitut, Institut für Organische Chemie (Direktor: Prof. Dr. Heyns) \\ der Universität Hamburg \\ Eingegangen am 21. April 1969
}

Mass-Spectrometric Detection of Drug Metabolites (Barbiturates, Noludar, Pyramidon) in Forensic Analysis. The mass-spectrometric identification of the metabolites isolated from biological material is discussed by means of the single mass spectra and of fragmentation patterns derived from them. In future, mass spectrography, mainly in combination with gas chromatography, will certainly more than hitherto contribute to the elucidation of many difficult problems of drug metabolism.

Zusammenfassung. Die massenspektrographische Identifizierung von aus biologischem Material isolierten Metabolitensubstanzen verschiedener Arzneimittel (Barbiturate, Noludar, Pyramidon) wird an Hand der einzelnen Massenspektren und ihrer daraus abgeleiteten Zerfallsschemata besprochen. In Zukunft wird die Massenspektrographie, vor allem kombiniert mit der Gas-Chromatographie, sicherlich mehr als bisher mit dazu beitragen, viele, häufig sehr differenzierte Probleme des Arzneimittelmetabolismus zu klären.

Wie in allen anderen Zweigen der analytischen Chemie besteht auch in der forensischen Chemie der Trend, den Nachweis toxikologisch relevanter Stoffe durch eindeutige, in kurzer Zeit durchführbare Analysenmethoden zu sichern. Einfache Farb- und Kristallfällungsreaktionen oder auch chromatographische $\mathrm{R}_{\mathrm{f}}$-bzw. Retentionswerte sind meist nicht ausreichend zur Definition einer forensisch isolierten Substanz. Mikroschmelzpunktbestimmungen und kristalloptische Analysenverfahren scheitern häufig an störenden Verunreinigungen, deren Entfernung, soweit möglich, zeitraubende Reinigungsverfahren erforderlich macht.
Bei vielen bekannten, toxisch wirksamen Arzneimittel- und Gift-Substanzen gelingt im allgemeinen eine Identifizierung mittels IR-Spektrum. Vereinzelt reicht aber auch ein IR-Spektrum nicht aus, um aus biologischem Material isolierte Giftspuren strukturell zu analysieren, insbesondere, wenn es sich um Metaboliten-Substanzen neuer Medikamente handelt, deren Stoffwechselabbauprodukte meist noch weitgehend unbekannt sind. Eine vergleichende Analyse durch Synthese der in Frage kommenden Substanzen verbietet sich häufig aus technischen Gründen, vielfach ist sie zu aufwendig. Da weiterhin meist nur minimale, dazu fast immer verunreinigte Substanz- 


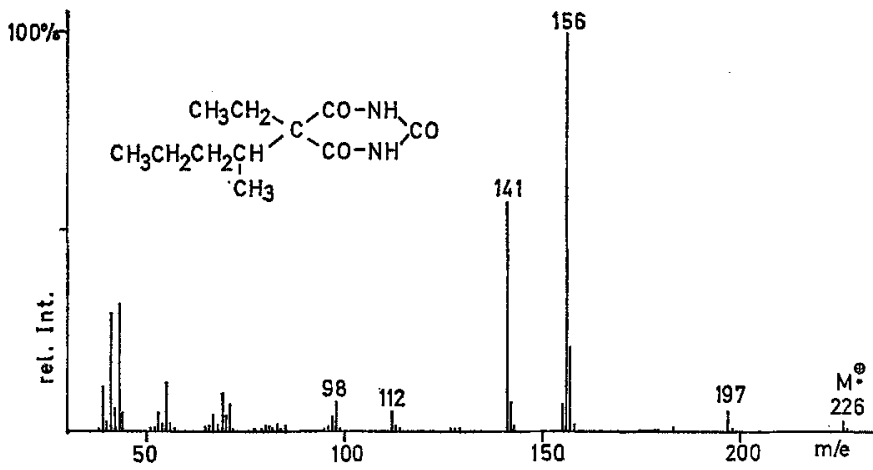
Abb. 1. Massenspektrum von Pentobarbital [5-Äthyl-
5-(1'-methylbutyl)-barbitursäure]

mengen als Ausgangsmaterial vorliegen, ist eine Identifizierung mit bisher üblichen analytischen Methoden nur in wenigen Fällen möglich. Hier bietet sich die Massenspektrometrie an, vor allem aufgrund ihres geringen Substanzbedarfes und ihrer nicht allzu

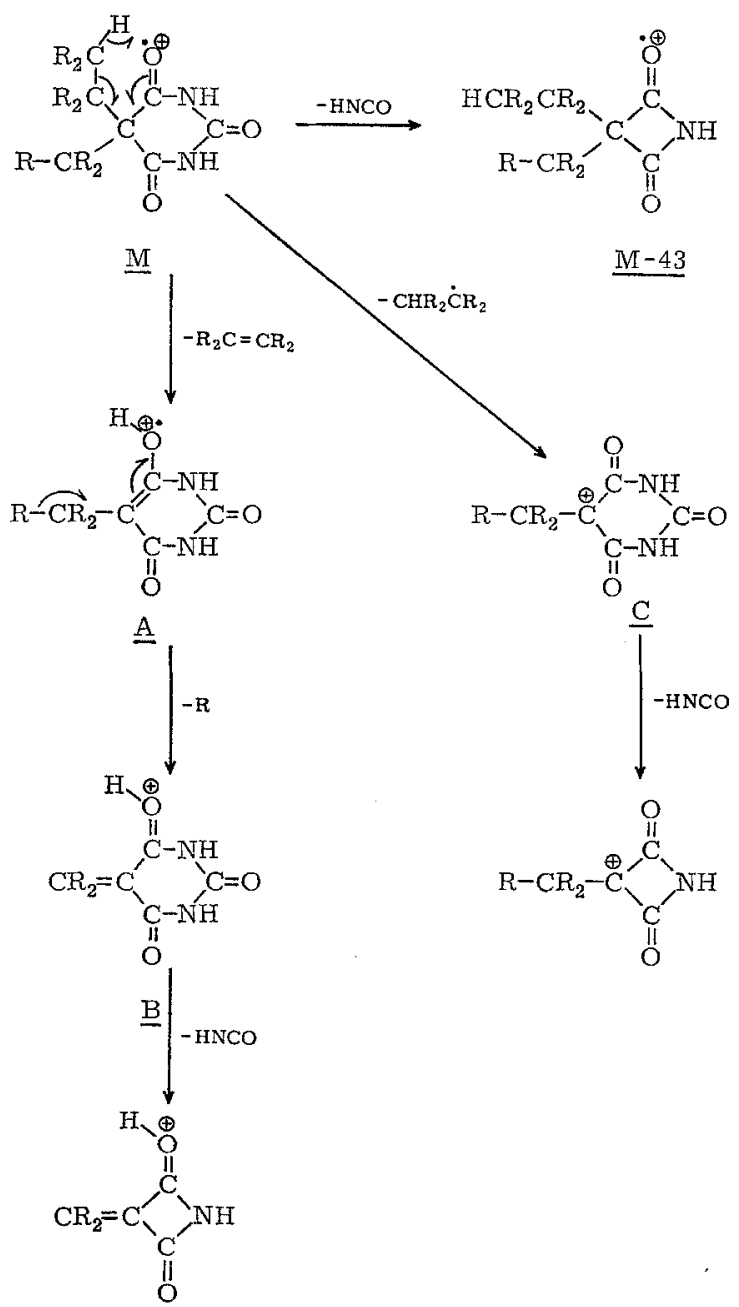

Abb. 2. Massenspektrometrisches Zerfallsschema für Barbiturate (allgemein) hohen Reinheitsanforderungen an die zu untersuchenden Stoffe.

In den letzten Jahren wurde mehrfach über den massenspektrometrischen Zerfallsmechanismus von Barbitursäurederivaten berichtet $[1,5-8]$.

Wie aus nachstehendem Massenspektrum und entsprechendem Zerfallsschema des Pentobarbitals ersichtlich ist - dies gilt analog auch für alle anderen Barbiturate - sind die Fragmentierungsvorgänge der beiden Substituenten am $\mathrm{C}_{5}$-Atom des Barbitursäureringes von besonderer Bedeutung, deren Elimination entweder als Radikal (beim Pentobarbital als $\mathrm{C}_{5} \mathrm{H}_{11}$ ) oder, vor allem, wenn zwei gesätt. Alkylsubstituenten vorhanden sind, über eine McLaffertyUmlagerung als Olefin (bei Pentobarbital als $\mathrm{C}_{5} \mathrm{H}_{10}$ Fragment) erfolgt. Beim Pentobarbital finden sich daher, neben dem kleinen Molekülpeak der Masse 226, entsprechende Ionen der Massen 197, 156 und 155 (s. Abb. 1 und 2).

Normalerweise wird, einer der Substituenten am $\mathrm{C}_{5}$ Atom ist fast ausnahmslos eine Äthylgruppe, immer die längere Seitenkette als Olefin abgespalten. Das zurückbleibende Molekülfragment besitzt dann die gleiche Massenzahl wie das Molekülion der Monoäthylbarbitursäure, differiert aber strulkturell und auch verhaltensmäBig infolge der Enolform dieses Ions. So spaltet die Ketoform im Rahmen einer McLafferty-Umlagerung die Äthylseitenkette als $\mathrm{C}_{2} \mathrm{H}_{4}$ Ion $a b$, während bei der Enolform vorwiegend ein Methylradikal abgespalten wird.

Diese Zerfallsreaktionen sind in Abb.3 noch einmal aufgeführt und konnten mit Erfolg benutzt werden, um die Struktur unbekannter Barbitursäure-Metaboliten massenspektrometrisch zu bestimmen.

Bei toxikologischen Routineuntersuchungen auf unveränderte Barbiturate wendet man die Massenspektrometrie im allgemeinen nicht an, da eine Identifizierung anhand von IR-Vergleichsspektren möglich ist. Andere Bedingungen liegen dagegen bei 


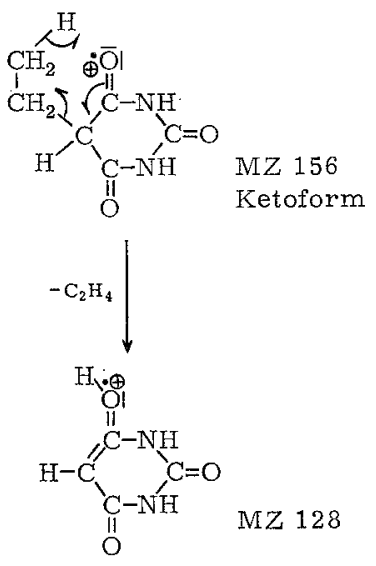

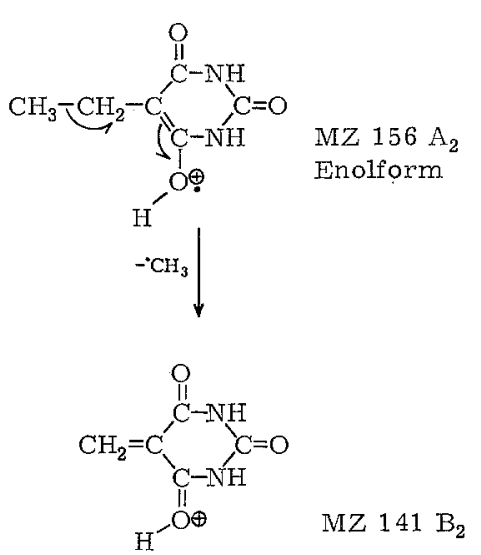

Abb. 3. Massenspektrometrische Zerfallsschemata der Monoäthylbarbitursäure (Keto- und Enolform) der Identifizierung von aus Organmaterial isolierten Metaboliten vor. Auch hier können nach präparativer dünnschicht-chromatographischer Auftrennung aus den einzelnen Fluorescenzauslöschungszonen nach Elution gut differenzierte und unterschiedliche IRSpektren erhalten werden, ohne daß es jedoch gelingt, eine sichere Bestimmung der molekularen Konfiguration durchzuführen. Um eine eindeutige Aussage zur Struktur der betreffenden Verbindung machen zu können, wurden einige dieser BarbituratMetaboliten einer massenspektrometrischen Analyse unterworfen $[1,4]$.

Bei der Untersuchung der Pentobarbital-Metaboliten ergab sich, daß nach präparativer dünnschichtchromatographischer Auftrennung der Barbituratfraktion bei einigen Vergiftungsfällen 6 Fluorescenzauslöschungszonen entstanden. 3 aus diesen Zonen isolierte Metabolitensubstanzen erwiesen sich massenspektrometrisch eindeutig als Barbiturate, 2 davon wiesen trotz unterschiedlicher $R_{\mathrm{f}}$-Werte im Dünnschicht-Chromatogramm fast völlig übereinstimmende Massenspektren (Abb.4) mit charakteristischen Peaks der Massenzahlen 98, 112, 141, 156 auf. Dies ist ein charakteristisches Zeichen dafür, daß es sich um Barbiturate mit Alkylsubstituenten, ähnlich wie bei der Muttersubstanz, handelt. Einer dieser Substituenten ist sicher, wie die großen Peaks mit den Massenzahlen 156 und 141 zeigen, als eine $\mathrm{C}_{2} \mathrm{H}_{5}$ Gruppe anzusehen. Die Bestimmung des 2. Substituenten gelang mit Hilfe des Molekülpeaks der Massenzahl 242. Aus der Differenz dieses Molekülpeaks zum Peak mit der Massenzahl 156 ist ersichtlich, daß aus dem Molekülion ein ungesättigtes Fragment der Masse 86 abgespalten wird. Dieses Molekülbruchstück kann nicht nur aus Kohlenstoff- und Wasserstoffatomen bestehen, sondern besitzt die Summenformel $\mathrm{C}_{5} \mathrm{H}_{10} \mathrm{O}$. Der kleine Peak im Massenspektrum mit der Zahl 224, entsprechend einer Abspaltung von $\mathrm{H}_{2} \mathrm{O}$, beweist wiederum, daß es sich bei der Sanerstoff-Funktion um eine Hydroxylgruppe handelt. Das Ion 224 besitzt demnach eine ungesättigte PentenylSeitenkette, die erfahrungsgemäß, wie bei anderen Barbituraten mit Alkylseitenketten, als Alkyl radikal beim weiteren Abbau abgespalten wird und ein Ion der Masse 155 entstehen läßt (vgl. Abb.5).

Die Stellung der Hydroyxlgruppe am $\mathrm{C}_{5}$-Substituenten ergibt sich aus den Peaks der Massenzahl 45 und 197, von denen der erstere auf die Bildung eines

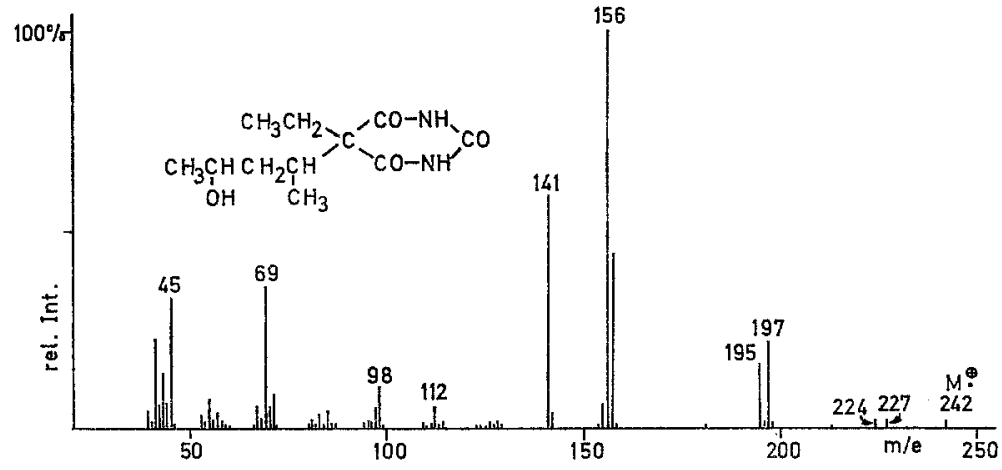

Abb. 4. Massenspektrum von Hydroxypentobarbital [5-Äthyl-5-(3-hydroxy-1-methylbutyl)-barbitursäure] 


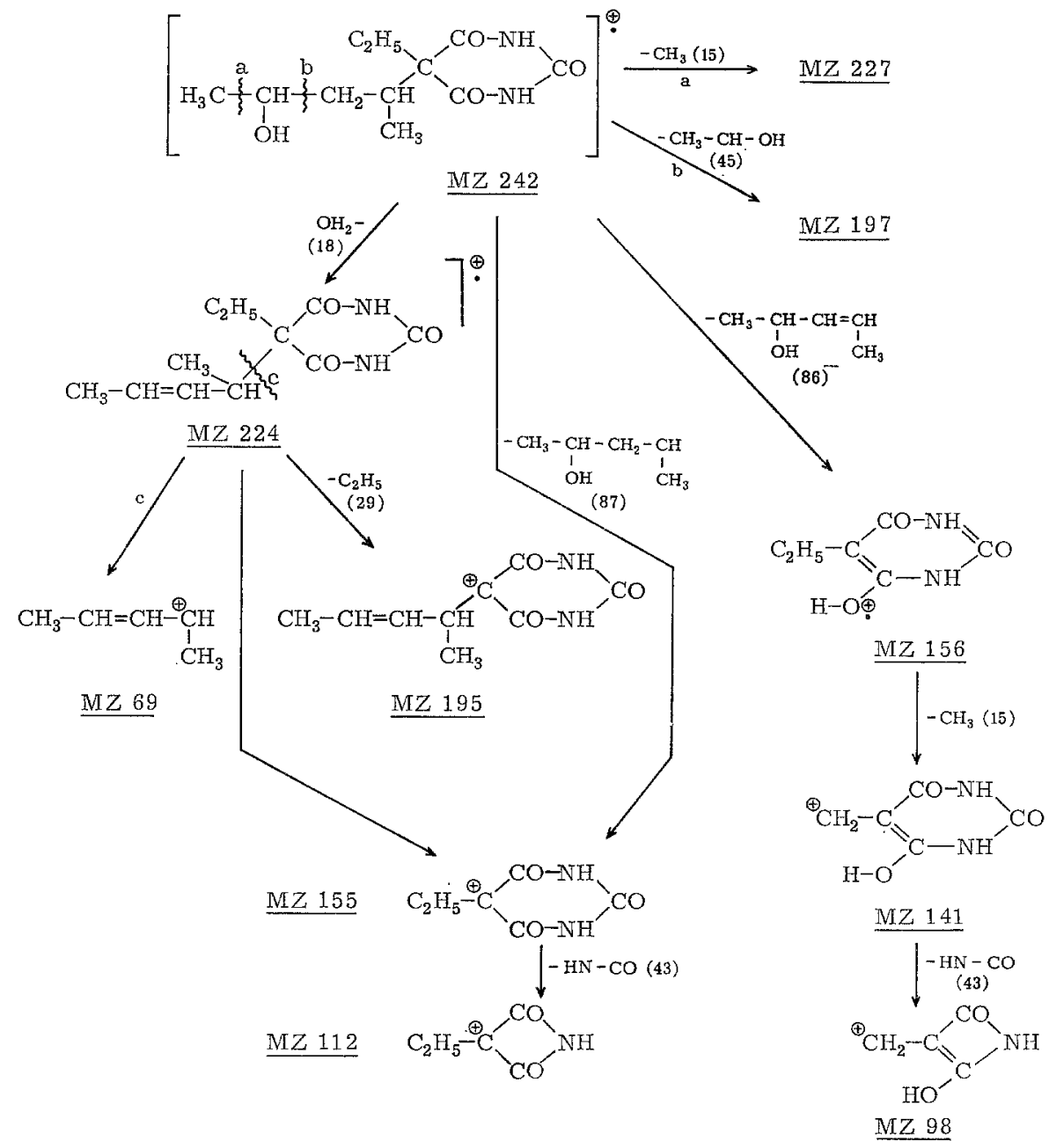

Abb. 5. Massenspektrometrisches Zerfallsschema für Hydroxypentobarbital

Oxeniumions mit der Massenzahl 45 hinweist. Die Abspaltung der gleichen Gruppe aus dem Molekülion führt dann zum Ion der Massenzahl 197. Demnach muß die Seitenkette des Pentobarbitals am $\mathrm{C}_{3}$-Atom hydroxyliert sein.<smiles>CCC(C(=O)O)(C(=O)NO)C(C)CC(C)(O)c1ccccc1</smiles><smiles>[R]C1C(C)C([R])C(C)C1C</smiles><smiles>[Z]C1C([R])C(C)C([R])C1C</smiles>

1-Form d-Form

Abb. 6. Diasteriomerie des Hydroxypentobarbitals
Durch diese Hydroxylierung am $\mathrm{C}_{3}$-Atom der Seitenkette entsteht ein neues zusätzliches Asymmetriezentrum (Abb.6). Es ist anzunehmen, da im Massenspektrum des zweiten Pentobarbitalmetaboliten alle vorher erwähnten Peaks in einer ähnlichen Verteilung der relativen Intensitäten vorhanden sind, daß es sich hier gleichfalls um einen am gleichen $\mathrm{C}_{3}$-Atom hydroxylierten Pentobarbital-Metaboliten handelt und infolgedessen beide Metaboliten Diasteriomere sind (s. Abb.4).

Mit Hilfe solcher massenspektrographischer Untersuchungen ist es gelungen, bei Vergiftungen mit Amobarbital, Evipan, Medomin, Phanodorm, Noctal und Luminal nach dünnschicht-chromatographischer Auftrennung der entsprechenden Barbiturat-Ausschüttelungsfraktionen $u$. a. folgende MetabolitenSubstanzen mit intaktem Barbitursäurering zu isolieren und massenspektrometrisch zu identifizieren [3]: 
Metaboliten von:

1. Amobarbital: . 5,5-Äthyl-(3'-hydroxyisoamyl)-barbitursäure.

2. Evipan: $\quad 5,5$-Cyclohexenonyl-methyl-1-methylbarbitursäure (Keto-Evipan).

3. Medomin: 5,5-Cycloheptenonyl-äthyl-barbitursäure, 5,5-Cyclohydroxyheptenyl-äthyl-barbitursäure.

4. Noctal: $\quad$ 5,5-Acetonyl-isopropyl-barbitursäure.

5. Phanodorm: 5,5-Cyelohexenonyläthyl-barbitursäure, 5,5-Cyclohydroxyhexenyl-äthyl-barbitursäure.

6. Phenobarbital: 5-Äthyl-5-(p-hydroxyphenyl)-barbitursäure.

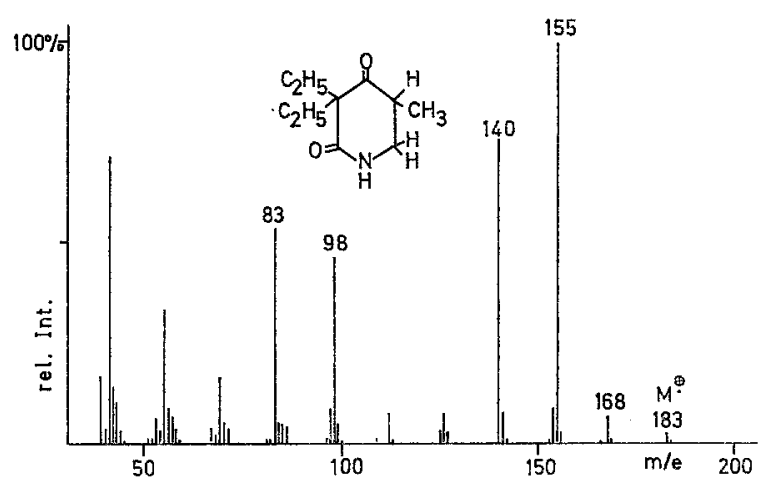

Abb. 7. Massenspektrum des Noludars (2,4-Dioxo-3,3-diäthyl-5-methylpiperidin)
Bei all diesen Metaboliten handelt es sich ausnahmslos um Verbindungen, bei denen, wie beim Pentobarbital, die Substituenten am $\mathrm{C}_{5}$-Atom oxydiert worden sind, und die daher Hydroxy- oder Ketogrup-<smiles>CCCCNC(=O)CC(C)C(=O)C(CC)(CC)CC</smiles>

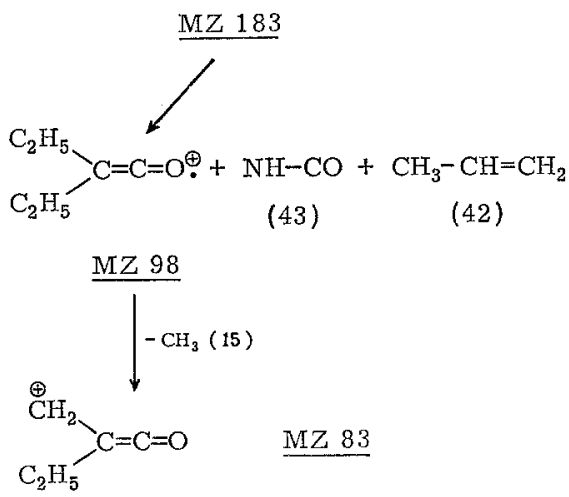

Abb. 9. Massenspektrometrisches Teilzerfallsschema für alle Noludarderivate ohne Doppelbindung im Ring<smiles>CCC1(CC)C(=O)NCC(C)C1=O</smiles>

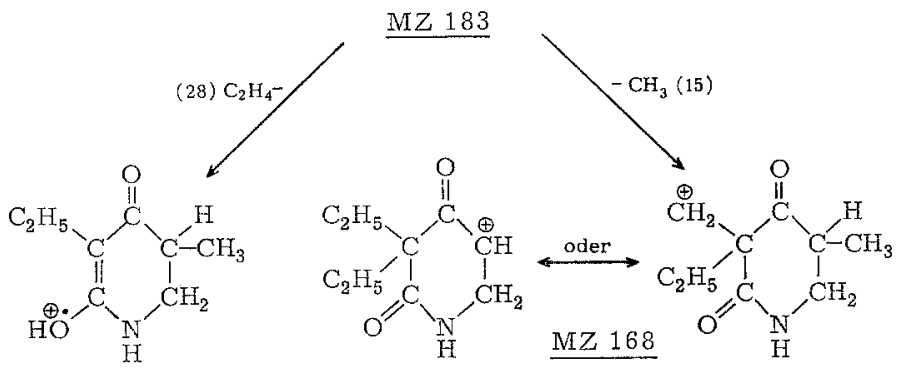

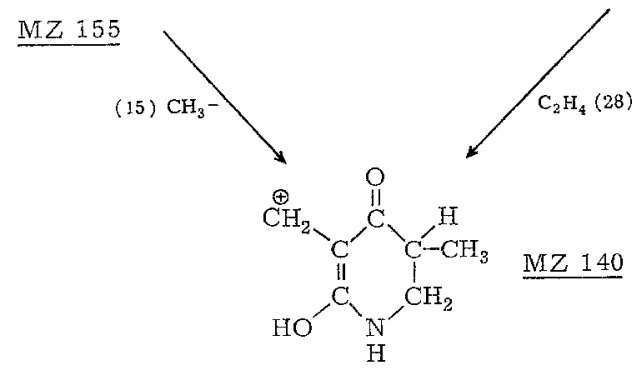

Abb. 8. Massenspektrometrisches Zerfallsschema von Noludar 
pen besitzen. Interessant war $น$. a. auch, daß trotz mehrfacher und auch mehrdimensionaler chromatographischer Auftrennung in diesen Fällen es nicht gelungen ist, einzelne Metabolitensubstanzen einwandfrei voneinander zu trennen. So ist aus den Massenspektren der Phanodorm- und auch MedominMetaboliten zu ersehen, daß in beiden Fällen ein Gemisch der Hydroxy- und Ketoverbindungen vorliegt. Dies erschwert eine IR-spektrophotometrische Identifizierung außerordentlich, stört jedoch den massenspektrometrischen Nachweis nicht wesentlich.<smiles>CCC1C(=O)NC(=O)C2(CC)CCC1CC2C</smiles>

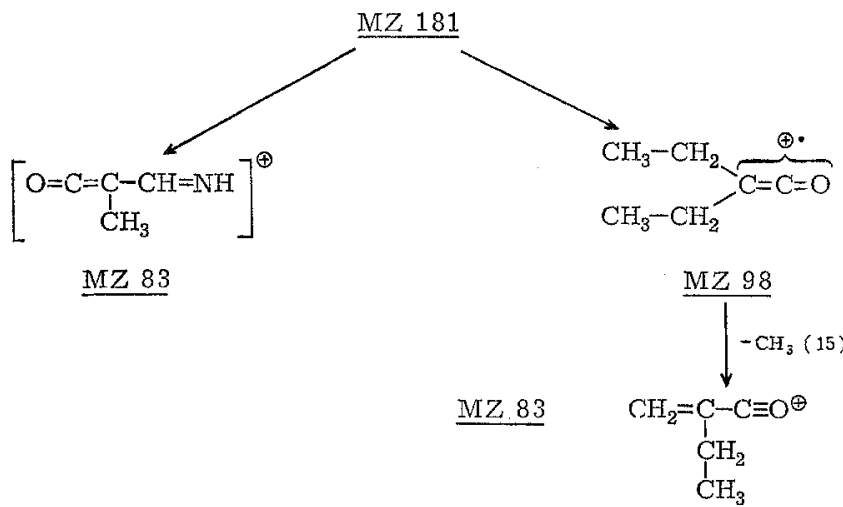

Abb. 10. Massenspektrometrisches Teilzerfallsschema für alle Noludarderivate mit Doppelbindung im Ring
In gleicher Weise können auch Noludar und seine Metaboliten massenspektrometrisch identifiziert werden, wie durch entsprechende Untersuchungen von Noludar-Metaboliten aus Urinproben bewußtlos gemachter und anschließend beraubter St. Pauli-Besucher festgestellt wurde $[2,3]$. Es gelang im Rahmen dieser Analysen, lediglich auf massenspektrometrischem Wege, ein neues Stoffwechselabbauprodukt des Noludars zu finden, das aus dem Urin eines solchen geschädigten St. Pauli-Besuchers isoliert und aufgrund seiner Herkunft als St. PauliMetabolit bezeichnet wurde.

Ähnlich wie die Barbiturate, weist das Massenspektrum des Noludars ebenfalls nur einen kleinen Molekülpeak auf (Abb.7). Entsprechend wird ein Substituent am quartären C-Atom durch eine McLafferty-Umlagerung zu einem Ion der Masse 155 abgespalten, gefolgt von der Abspaltung einer $\mathrm{Me}$ thylgruppe an dem 2. Substituenten zum Ion 140 (Abb.8). Die Fragmentionen der Masse 98 und 83 sind aufgrund unserer Untersuchungen als charakteristisch für das Dioxopiperidingerüst anzusehen. Die Bildung dieser Ionen zeigt Abb.9. Durch eine cyclische Elektronenverschiebung im Ring entsteht ein Ion 98 mit der Struktur eines Dialkylketons, aus dem dann eine Methylgruppe zum Ion 83 abgespalten wird. Die gleiche Reaktion findet sich auch bei Noludar-Verbindungen mit einer Doppelbindung im Ring (Abb. 10).

Das Massenspektrum des unbekannten Metaboliten des Noludars besitzt gleichfalls sehr große Peaks der Massen 98 und 83, so daß diese Verbindung sicher als ein 2,4-Dioxo-3,3-diäthylpiperidinderivat anzusehen ist (Abb.11). Der Peak der Massenzahl 181 würde dem Molekülion des 2,4-Dioxo-3,3-diäthyl-

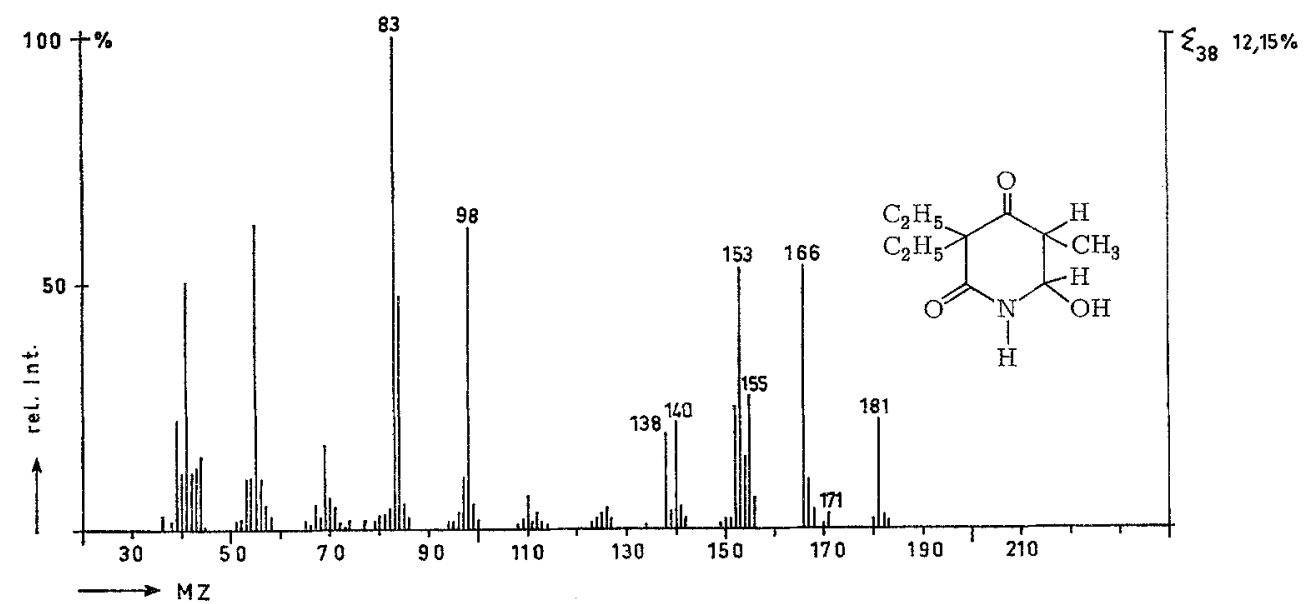

Abb. 11. Massenspektrum des St. Pauli-Noludarmetaboliten (2,4-Dioxo-3,3-diäthyl-5-methyl-6-hydroxypiperidin) 
5-methyltetrahydropyridin entsprechen. Der kleine Peak bei 171 und noch einige weitere Einzelheiten des Spektrums zeigen jedoch, daß dieses Ion nicht dem Molekülpeak entspricht, sondern die Verbindung ein Molekulargewicht von 199 besitzt und als ein 2,4Dioxo-3,3-diäthyl-5-methyl-6-hydroxylpiperidin anzusehen ist. Der Peak 181 ist durch eine schnelle Albspaltung von Wasser aus der Hydroxylgruppe am $\mathrm{C}_{6}$-Atom des Noludarringes entstanden, so daß, wie

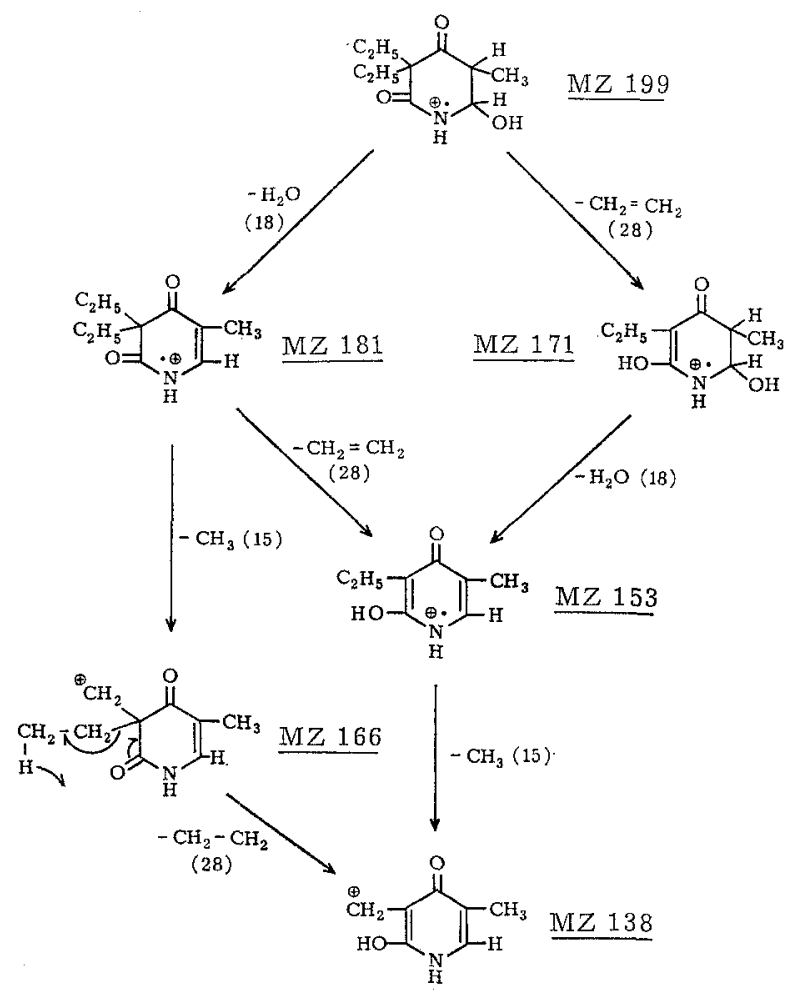

Abb. 12. Massenspektrometrisches Zerfallsschema des St. Pauli-Noludarmetaboliten gesagt, der Molekülpeak mit der Massenzahl 199 nicht erfaßt wird (Abb.12). Der Peak mit der Massenzahl 171 entsteht durch Abspaltung einer der Äthylgruppen als Äthylen am $\mathrm{C}_{3}$-Atom des intakten Molekülions [3].

Weiter wurde versucht, Pyramidon-Metaboliten auf massenspektrometrischem Wege zu identifizieren.

Das Massenspektrum des Pyramidons zeigt neben seinem Molekülpeak mit der Massenzahl 231 im oberen Massenbereich keine charakteristischen Peaks (Abb. 13). Offensichtlich zerfällt das Molekülion dieser Ver. bindung sehr leicht unter Spaltung des Pyrazolinonringes (Abb.14). Dabei werden die $\mathrm{N}$-N-Bindung und die Bindung neben der Carboxylgruppe gespalten, so daB zwei Fragmente der Masse 112 und 119 entstehen. Beide Fragmente werden in geringer Menge als Ionen beobachtet. Während aber für das Fragment mit der Massenzahl 119 die Struktur des relativ stabilen Phenylisocyanats formuliert werden kann, läßt sich für das Ion der Masse 112 keine stabile Struktur angeben. Dieses Ion zerfällt daher schnell weiter unter Bildung von Ionen der Masse 111, 97 und 56 (s. Abb. 14). Die elementare Zusammensetzung wurde durch Hochauflösung gesichert, die Verknüpfung der einzelnen Reaktionsschritte läßt sich durch metastabile Ionen nachweisen. Man erkennt, daß die Ionen vorwiegend stickstoffhaltige Fragmente mit konjugierten Doppelbindungen sind.

Als Stoffwechselprodukte des Pyramidons sind u. a. 4-Amino-antipyrin, 4-Acetamido-antipyrin, Methylrubazonsäure und Rubazonsäure bekannt. Diese Verbindungen wurden bei einigen Vergiftungsfällen isoliert und massenspektrometrisch identifiziert. Die Massenspektren des 4-Amino-antipyrins (Abb.15)

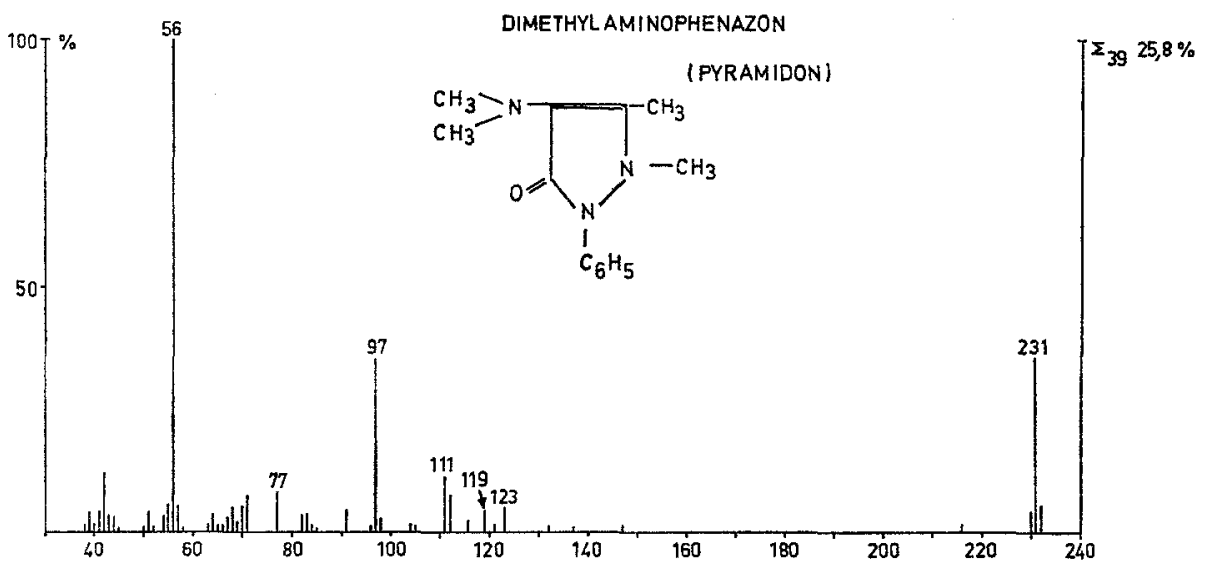

Abb. 13. Massenspektrum von Pyramidon (Dimethylaminophenyldimethyl-pyrazolon) 
<smiles>Cc1c(N(C)C)c(=O)n(-c2ccccc2)n1C</smiles>

$\mathrm{O}=\mathrm{C} \stackrel{\oplus}{=} \stackrel{\bullet}{\mathrm{N}}-\mathrm{C}_{6} \mathrm{H}_{5}$

MZ 119

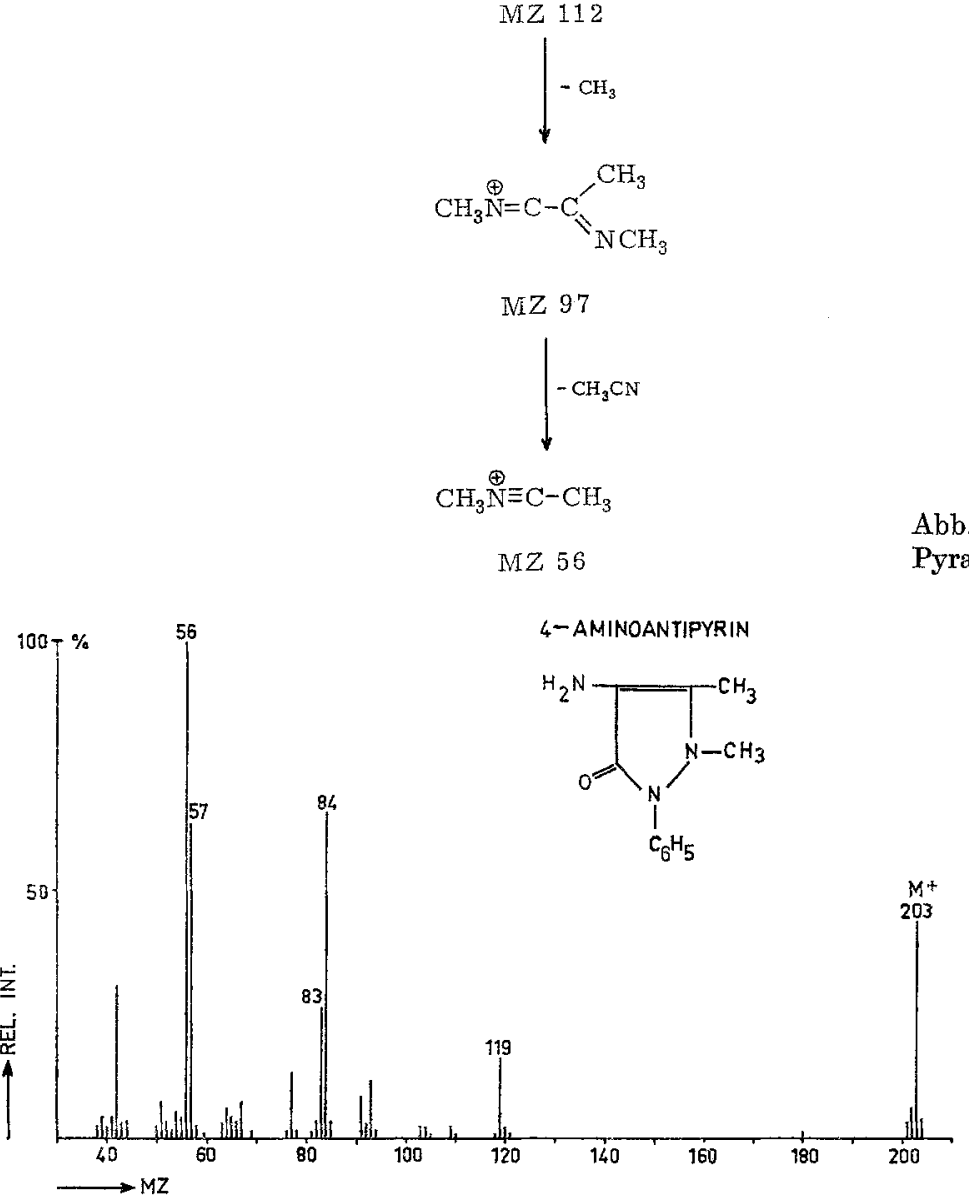

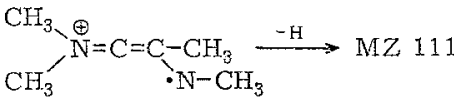

MZ 231

$-(119)$

112

bb. 14. Massenspektrometrisches Zerfallsschema von Pyramidon
Abb. 15. Massenspektrum von 4-Aminoantipyrin (1-Phenyl-2,3-dimethyl-4-aminopyrazolon)

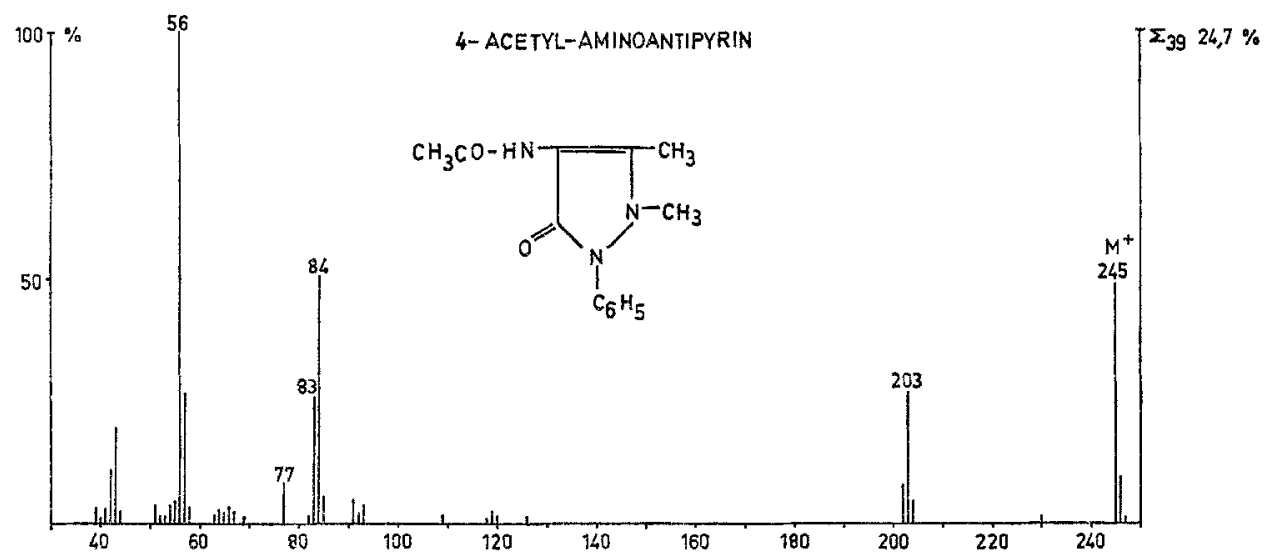

Abb. 16. Massenspektrum von 4-Acetyl-aminoantipyrin (1-Phenyl-2,3-dimethyl-4-acetaminopyrazolon) 

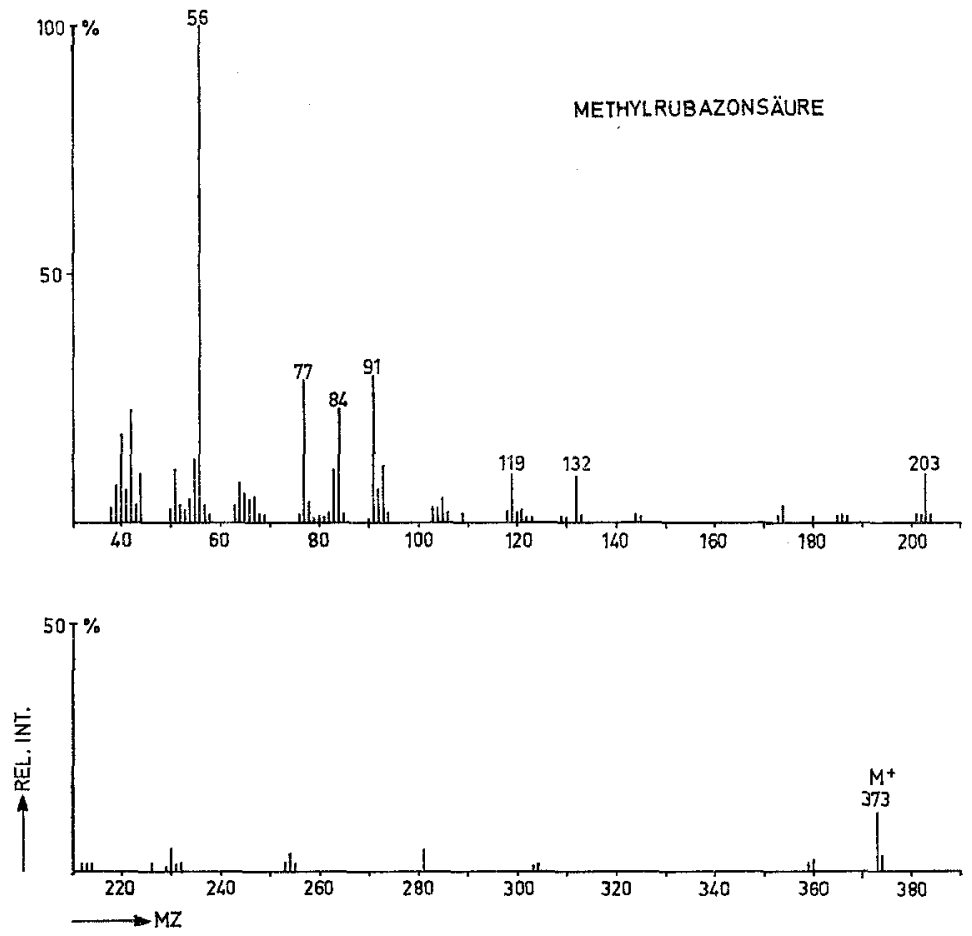

Abb. 17. Massenspektrum von Methylrubazon-

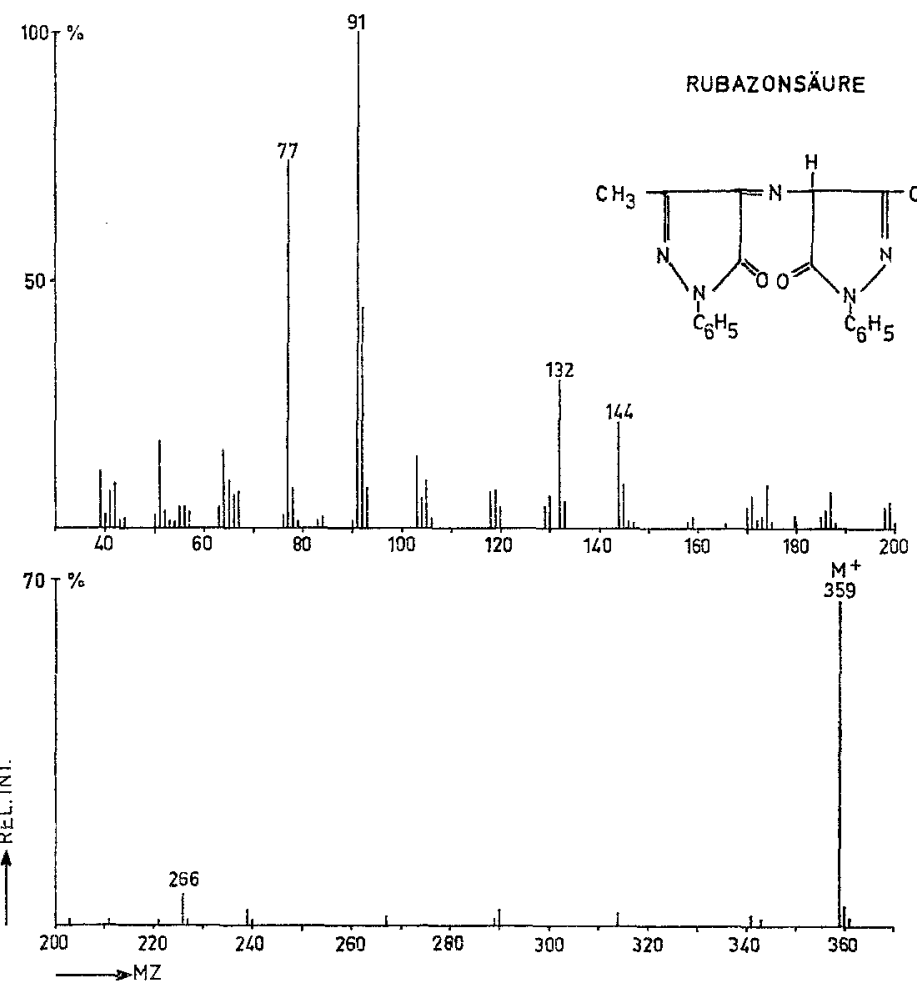
säure

Abb. 18. Massenspektrum von Rubazonsäure

und des 4-Acetylderivates (Abb.16) sind analog dem Spektrum des Pyramidons zu deuten. Auch zwischen dem Massenspektrum der Methylrubazonsäure (Abb.17) und den anderen $\Delta^{3}$-Pyrazolinonen besteht noch enge Verwandtschaft, während Rubazonsäure selbst als $\Delta^{2}$-Pyrazolinonderivat einem völlig anderen massenspektrometrischen Zerfallsmechanismus unterliegt (Abb. 18). 
Die vorstehenden Ergebnisse weisen darauf hin, daß die Massenspektrometrie, vor allem auch in Verbindung mit der Gas-Chromatographie, zukünftig im Rahmen der forensisch-chemischen Analyse wesentlich an Bedeutung gewinnen wird. Mit ihrer Hilfe werden sicher viele, zur Zeit noch strittige Probleme des Gift- und Arzneimittelstoffwechsels gelöst werden können. Für die Konstitutionsermittlung und Identifizierung von Metabolitensubstanzen ist sie bereits schon jetzt als Methode der Wahl anzusehen.

\section{Literatur}

1. Arnold, W.: Habilationsschrift, Universität Hamburg 1967.

2. - Kriminalistik 20, 363 (1966).
3. Arnold, W., u. H. F. Grätzmacher: Deut. Z. Ges. Gerichtl. Med. 65, 44-60 (1969).

4. - - Die Identifizierung von Barbiturat-Metaboliten mittels Massenspektrometrie und Infrarotspektrum. (Arch. Toxikol. im Druck).

5. Costopanagiotis, A., u. H. Budzikiewicz: Monatsh. Chem. 96, 1800-1808 (1965).

6. Grützmacher, H. F.: Deut. Apotheker-Ztg. 106, 377-382 (1966).

7. -, u. W. Arnold: Tetrahedron Letters 13, 1365-1374 (1966).

8. - - Vortrag, Chemie-Dozenten-Tagung, Würzburg 1966

Doz. Dr. med. Dr. rer. nat. Wolfgang Arnold

2000 Hamburg-Lokstedt, Butenfeld 34

Doz. Dr. H. F. Grützmacher

2000 Hamburg 13, Papendamm 6

\title{
Microdosage direct du bore dans les plantes par la méthode fluorimétrique à l'hydroxy-2-méthoxy-4-chloro-4'-benzophénone (HMCB)
}

\author{
D. Monnier, B. Liebich et M. Marcantonatos \\ Laboratoires de Chimie Analytique, Université de Genève (Suisse) \\ Reçu le 2 mai, 1969
}

Direct Microdetermination of Boron in Plants by a Fluorimetric Method Using Hydroxy-2-methoxy-4-chloro$4^{\prime}$-benzophenone (HMCB). The method is proposed for the direct determination of submicrotraces of boron in as little as about 0.2 to $1 \mathrm{mg}$ of plant material. A mixture of concentrated sulphuric acid and hydrogen peroxide $(30 \%)$ at $200^{\circ} \mathrm{C}$ is employed for the mineralisation. There is no loss of boron even for quantities in the nanogram range. For $30 \mathrm{ng}$ of boron fluorine interferes only for ratios $\mathrm{F} / \mathrm{B}(\mathrm{w} / \mathrm{w}) 100$, but analytical ourves - while less sensitive-are even satisfactory for higher ratios. Several applications are given.

Résumé. La méthode fluorimétrique à l'hydroxy-2-méthoxy-4-chloro-4'-benzophénone (HMCB) est appliquée au dosage direct de traces de bore dans des échantillons végétaux de 0,2 à $1 \mathrm{mg}$. La minéralisation d'effectue par un mélange d'acide sulfurique concentré et de perhydrol $(30 \%)$ à $200^{\circ} \mathrm{C}$. Pour des quantités de bore de l'ordre du nanogramme, ce procédé n'entraîne pas de pertes. Pour 30 ng de bore, les fluorures n'interfèrent que pour $\mathrm{F} / \mathrm{B}(\mathrm{P} / \mathrm{P})>100$. Pour des rapports supérieurs, les résultats, quoique moins sensibles, permettent toutefois d'établir des courbes d'étalonnage satisfaisantes. Des exemples d'applications sont donnés.

Le fait que le bore est indispensable au métabolisme des plantes supérieures est maintenant définitivement acquis. Or, la concentration de cet élément est souvent très variable d'une partie à l'autre d'une plante donnée. Il serait donc utile de pouvoir disposer d'une méthode analytique permettant d'effectuer des dosages sur des prises très faibles d'échantillons et pouvant, ainsi, fournir des ren- seignements précis sur la distribution du bore dans un végétal donné.

La méthode fluorimétrique à l'HMCB [5] que nous proposons permet d'atteindre ce but. En effet, grâce à sa sélectivité et à sa sensibilité élevée, il est possible de doser avec une précision satisfaisante des nanogrammes de bore contenus dans des prises de $0,2 \grave{\mathrm{a}}$ 1,2 mg (poids sec) d'échantillon végétal. 